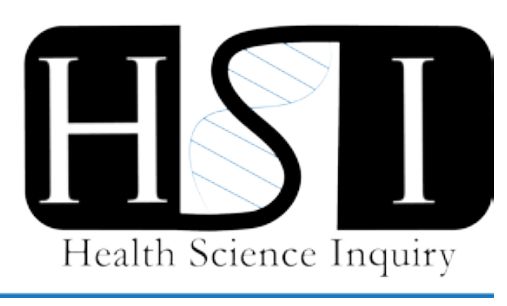

\title{
Exploring immersive technologies: the potential for innovation in whiplash research
}

Michael Lukacs, Shahan Salim

Faculty of Health Sciences, University of Western Ontario

Whiplash-acquired disorders (WAD) are some of the most common injuries acquired as a result of a motor vehicle collision (MVC) [1]. WAD and its associated sequelae often present as neck pain, musculoskeletal deficits or neurological signs [2]. It has been estimated that of the individuals who sustain such an injury, 20 to 50 percent will experience chronic alterations of their activities of daily living as much as 1 year later [1,3]. WAD also imposes a large economic burden on the healthcare system, which leads to billions of dollars spent every year for rehabilitation of these injuries, not including the time spent away from work [4]. WAD represents a huge hardship for sufferers, their caregivers as well as their employers. Given these burdens, whiplash research requires innovation which could be accomplished via virtual-reality (VR) and augmented reality $(A R)$ in order to better understand it.

Despite the socioeconomic burden of WAD, it is unclear as to why some individuals are more predisposed to WAD, or how acute to chronic WAD occurs $[3,4,5]$. Many traditional models have looked at the biomechanical characteristics of a MVC (e.g., speed/direction of impact, awareness of impending collision) in order to explain the development of WAD $[3,4]$. The current consensus is that the associations between the biomechanical characteristics of traumatic events, such as a car crash, and clinical symptoms (headache, neck pain) are weak or poorly understood $[3,4,5]$. As a result, clinicians struggle to explain to patients how they developed their symptoms.

Consequently, new mechanistic models have been developed to explain the curiosity that is WAD. It is believed that the onset of many chronic health conditions, such as WAD or post-traumatic distress, can be linked back to a maladaptive stress reaction to trauma [6]. Other mechanistic models now include both the reaction to a traumatic event (i.e., MVC), as well as pre-existing traits of the individual, both organic (genetics) and non-organic (socioeconomic status, psychological factors) in nature $[3,5]$. It has also been proposed that approximately $20 \%$ of individuals subjected to a 'placebo' MVC will exhibit WAD-like symptoms, despite no physical car collision occurring [4]. This may be due to perceived psychological stress such as anxiety of a perceived impact, but at this point it remains unclear [4]. These models are difficult to empirically assess, as most are in the theoretical stage because it is not ethical to place individuals in real-world car crashes. Furthermore, it is difficult to experimentally manipulate the parameters of a traumatic event under natural circumstances, including capturing of the stress/startle reactions of normal humans in real-world car crashes.

Given the advent of immersive technologies such as VR and $A R$, the study of stress/startle responses in traumatic incidents could be possible. VR has been described as an interface where the user interacts with 3-D objects with real time feedback [7]. AR can be described as the digital overlap of real space which can be manipulated [7]. VR and AR technologies have had increasing use in the world of medicine, from revolutionizing the ways surgeries are performed to changing the education of anatomy [7]. In clinical applications, VR has been documented to be effective in both the assessment and intervention of range of motion for the cervical spine [8]. In these applications, VR has proven effective as it allows for capture of intended variables in a simulated environment, such that responses are more ecological in nature, as if they happened in the real world [8]. In the field of phobia research, VR has shown to be quite efficacious in producing simulated environments resembling real-world ones, with the goal of promoting behavioural change [9].

While VR has been used for pain assessments and kinematic measures of the neck, to date no investigations have used VR to study the psychosocial domains or possible mechanisms of WAD $[8,10]$. VR or AR technologies could be radical in their ability to give subjects the perception of driving or being a passenger in a MVC, despite not experiencing an actual car crash. A VR road collision simulator could help develop inroads to why whiplash injuries occur through exploration of the psychosocial domains of WAD without any biomechanical input. In this manner, many of the mechanisms described above to explain WAD can be investigated in a robust fashion. The results of these studies could provide potential new explanations for WAD as well as inform future interventions. 
Applications of immersive technologies such as VR and $A R$ are well documented and appear poised to continue progressing $[7,8,9,10]$. The use of these technologies allows for the types of research that have not been possible to conduct previously and exploration of purely theoretical mechanistic models in WAD research $[3,4,5,6]$. Truly, the pieces are present for dedicated researchers to help understand the problem that is WAD, and aid in decreasing the burden of this common condition.

\section{References}

[1] Carroll, L. J., Hogg-Johnson, S., Côté, P., van der Velde, G., Holm, L. W., Carragee, E. J., Hurwitz, E. L., Peloso, P. M., Cassidy, J. D., Guzman, J., Nordin, M. (2008) Course and prognostic factors for neck pain in workers. European Spine Journal, 17 (1), 93-100.

[2] loppolo, F., Rizzo, R. S. (2014) Epidemiology of whiplash-associated disorders. In: Whiplash Injuries, 2014, 13-16. Springer, Milano.

[3] Walton, D. M., Elliott, J. M. (2017) An integrated model of chronic whiplash-associated disorder. Journal of orthopaedic \& sports physical therapy, 47 (7), 462471.

[4] Castro, W. H., Meyer, S. J., Becke, M. E., Nentwig, C. G., Hein, M. F., Ercan, B. I., Thomann, S., Wessels,
U., Du Chesne, A. E. (2001) No stress-no whiplash?. International journal of legal medicine, 114 (6), 316322.

[5] Evans RW. Persistent Post-Traumatic Headache, Postconcussion Syndrome, and Whiplash Injuries: The Evidence for a Non-Traumatic Basis With an Historical Review. Headache: The Journal of Head and Face Pain, 50 (4), 716-724.

[6] Alschuler, K. N., Kratz, A. L., Ehde, D. M. (2016) Resilience and vulnerability in individuals with chronic pain and physical disability. Rehabilitation psychology, $61(1), 7$.

[7] Müller-Wittig, W. (2011) Virtual reality in medicine. In: Springer Handbook of Medical Technology, 2011, 1167-1186. Springer, Berlin.

[8] Sarig-Bahat, H., Weiss, P. L., Laufer, Y. (2009) Cervical motion assessment using virtual reality. Spine, 34 (10), 1018-1024.

[9] Morina, N., ljntema, H., Meyerbröker, K., Emmelkamp, P. M. (2015) Can virtual reality exposure therapy gains be generalized to real-life? A metaanalysis of studies applying behavioral assessments. Behaviour research and therapy, 74, 18-24.

[10] Sarig-Bahat, H., Weiss, P. L., Laufer, Y. (2010) Neck pain assessment in a virtual environment. Spine, 35 (4), E105-E112. 


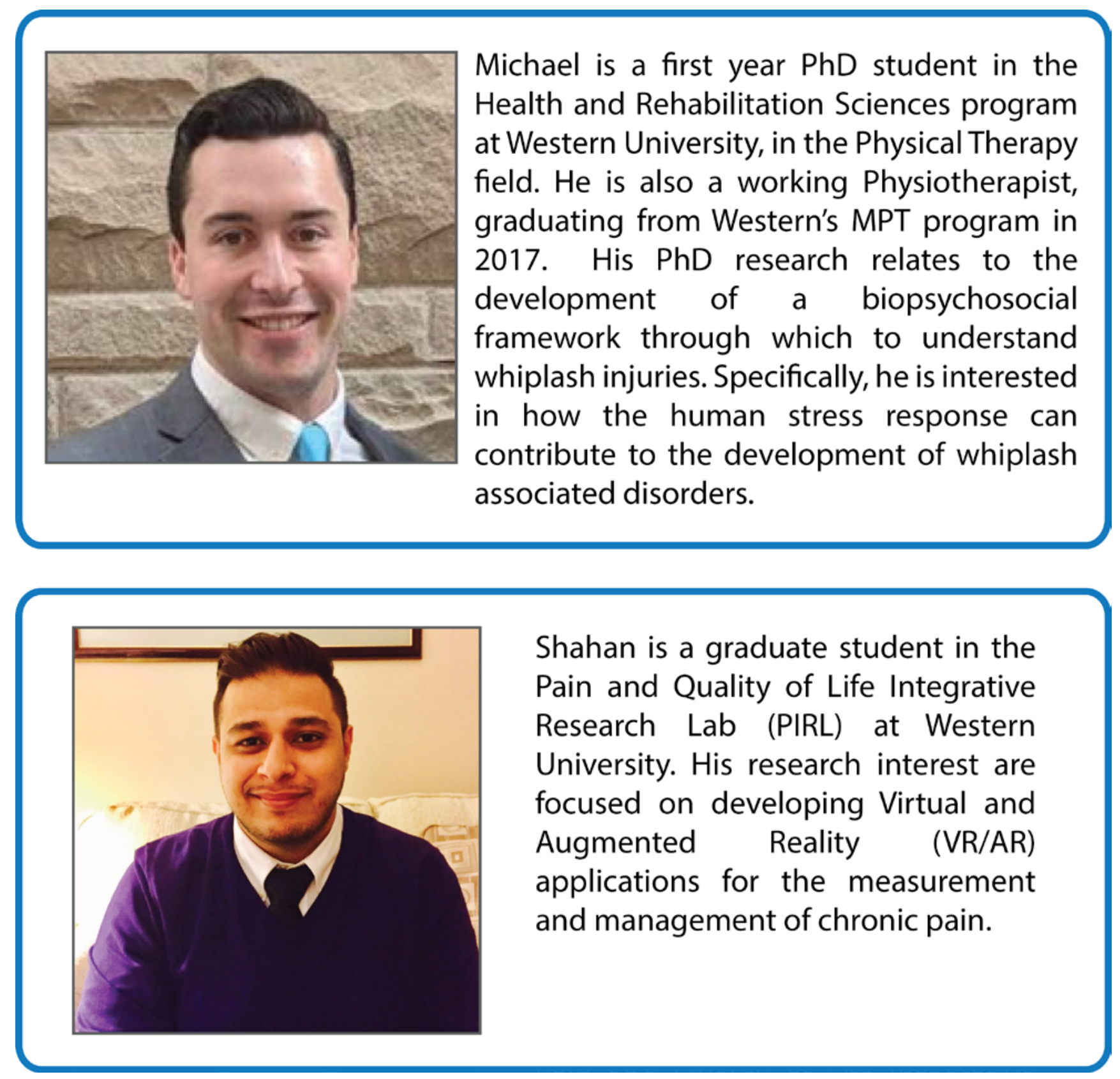

